\title{
Rise of correlated dislocations in nanotwinned metals against fatigue
}

\author{
Yongjun Tian
}

When a metal is subject to cyclic loading with cyclic stress values much lower than its tensile strength, fatigue occurs due to accumulative and irreversible damages developed in the microstructure, causing service failure of the metal workpiece or even fatal disaster in the worst cases. To develop materials with higher fatigue limit and longer fatigue life relies on reducing or suppressing these structural damages during cyclic deformation, and microstructure design via a nanostructuring strategy provides a possibility to fulfill this goal [1]. For example, ultrafine-grained (UFG) and nanocrystalline (NG) metals usually exhibit much improved fatigue life in the high cycle fatigue (HCF) regime compared with their conventional coarse grain (CG) counterparts [2]. Still, the fatigue life of UFG and NG metals in the low cycle fatigue (LCF) regime is much shorter than that of CG counterparts due to degraded ductility [3,4]. Meanwhile, great research endeavors have been devoted to strengthening metals by means of engineering coherent twin boundaries (TBs), which can work as barriers for dislocation motion and accommodate dislocations simultaneously $[5,6]$. However, systemically evaluation of fatigue properties of nanotwinned (NT) metals is inadequate due to the very limited sample thickness [7-9].

To solve this difficulty, Lu's group from Shenyang National Laboratory for Materials Science, Chinese Academy of Science synthesized bulk (thickness $\geq 3 \mathrm{~mm}$ ) high-purity $\mathrm{Cu}$ plates with nanoscale growth twins by using a direct-current electrodeposition method, and conducted uniaxial symmetric tension-compression cyclic deformation tests (the loading axis is approximately parallel to the twin planes), with stepwise increasing and decreasing plastic strain amplitude $\Delta \varepsilon_{\mathrm{pl}} / 2$ [10]. Crosssectional scanning electron microscopy (SEM) and transmission electron microscopy (TEM) observations on the NT-Cu samples revealed columnar grains separated by sharp grain boundaries (Fig. 1a) and high-density nanotwin structures in the columnar grains with TBs preferentially parallel to the deposition plane (Fig. 1b), respectively. The deformation tests showed the cyclic stress response quickly reaches a saturation value at each $\Delta \varepsilon_{\mathrm{pl}} / 2$ with a one-to-one correspondence between stress and strain amplitude (Fig. 1e, f). Their results clearly indicate NT-Cu exhibits a unique, stable and history-independent cyclic response, which is in stark contrast with CG-Cu and UFG-Cu showing unstable and history-dependent cyclic responses (either hardening or softening).

In collaboration with Gao's group from Brown University, Lu et al. elucidated the deformation mechanisms of the unique cyclic response of NT-Cu on the base of atomistic simulations and microstructure analyses. Due to highly oriented nanotwins within the columnar grains and the loading directions approximately parallel to the TBs, only a single slip system with slip plane inclined to TBs was active during the cyclic tests, leading to highly organized correlated necklace dislocations (CNDs) across multiple TBs formed in NT-Cu. No strain localization occurs inside of the grain, and dislocation locks and entanglements are greatly suppressed. Under cyclic loading, parallel CNDs within each grain move collectively back and forth along TBs without accumulating damages to the nanotwins (See Fig. 1c, d for the microstructure of NT-Cu after cyclic loading). The delocalized cyclic deformation mechanism in NT-Cu is fundamentally distinct from the conventional strain localizing mechanisms associated with irreversible microstructural damage in single-crystal, CG, UFG, and NG metals.

Clearly the stable and history independent cyclic response stems from the highly oriented nanotwinned structure. Combined with the advantages of TBs at nanoscales for achieving high strength meanwhile maintaining ductility and work hardening, superb fatigue

State Key Laboratory of Metastable Materials Science and Technology, Yanshan University, Qinhuangdao 066004, China

Email: fhcl@ysu.edu.cn 

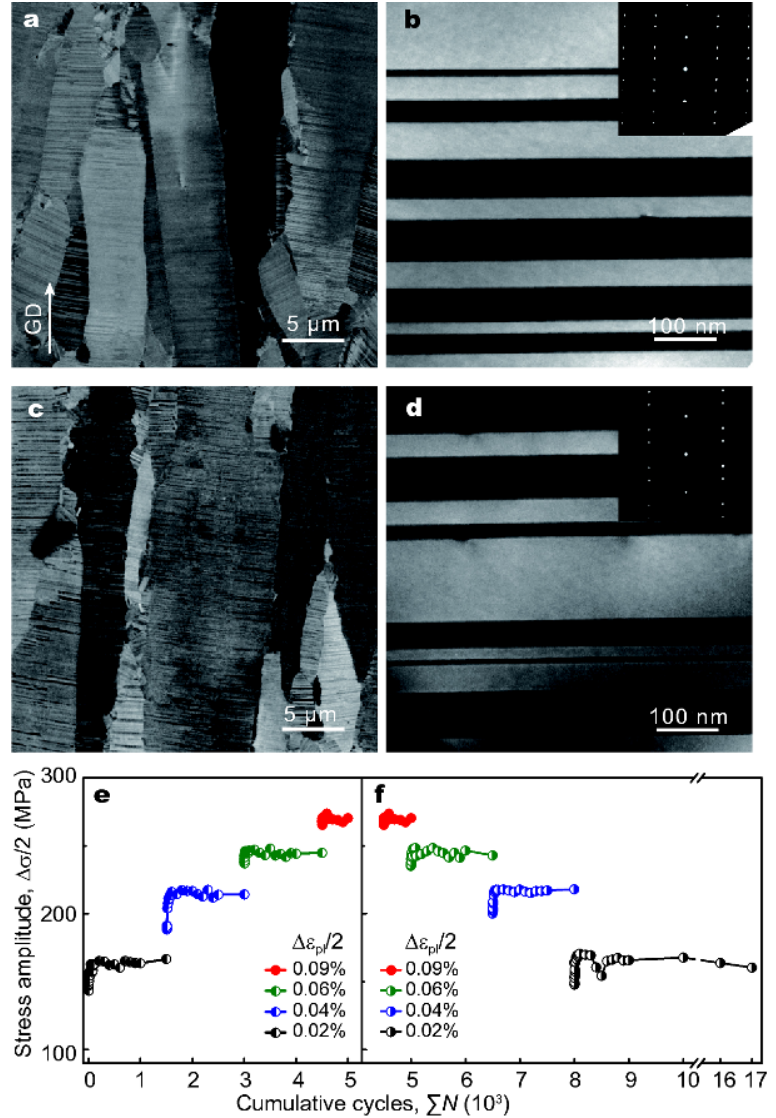

Figure 1 Microstructure and cyclic deformation behavior of NT-Cu. (a) Cross-sectional SEM and (b) TEM before cyclic deformation. (c) Crosssectional SEM and (d) TEM after 17,000 loading cycles. (e) and (f) Cyclic stress response $\Delta \sigma / 2$ as a function of the cumulative loading cycles $\Sigma N$ for NT-Cu cyclically deformed in increasing and decreasing sequences of plastic strain amplitudes, respectively. Insets in (b) and (d) show selected area electron diffraction patterns of nanoscale twins [10]. Copyright 2017, Macmillan Publishers Limited, part of Springer Nature. properties can be achieved in nanotwinned metals, such as long fatigue life in the LCF regime and high fatigue limit in the HCF regime. Their findings pave a new route to more fatigue-resistant metals through tailor-designed microstructure. For practical applications of this excellence, economical and efficient methods to produce bulk metals or alloys with highly oriented nanotwins are urgently needed.

Received 21 November 2017; accepted 23 November 2017; published online 14 December 2017

1 Lu K. Stabilizing nanostructures in metals using grain and twin boundary architectures. Nat Rev Mater, 2016, 1: 16019

2 Meyers MA, Mishra A, Benson DJ. Mechanical properties of nanocrystalline materials. Prog Mater Sci, 2006, 51: 427-556

3 Kwan CCF, Wang Z. Cyclic deformation behavior of ultra-fine grained copper processed by accumulative roll-bonding. Procedia Eng, 2010, 2: 101-110

4 Mughrabi H, Höppel HW. Cyclic deformation and fatigue properties of very fine-grained metals and alloys. Int J Fatigue, 2010, 32: 1413-1427

5 Christian JW, Mahajan S. Deformation twinning. Prog Mater Sci, 1995, 39: 1-157

$6 \mathrm{Lu} \mathrm{K}, \mathrm{Lu} \mathrm{L}$, Suresh S. Strengthening materials by engineering coherent internal boundaries at the nanoscale. Science, 2009, 324: 349-352

7 Shute CJ, Myers BD, Xie S, et al. Detwinning, damage and crack initiation during cyclic loading of $\mathrm{Cu}$ samples containing aligned nanotwins. Acta Mater, 2011, 59: 4569-4577

8 Pan QS, Lu QH, Lu L. Fatigue behavior of columnar-grained $\mathrm{Cu}$ with preferentially oriented nanoscale twins. Acta Mater, 2013, 61: 1383-1393

9 Pan QS, Lu L. Strain-controlled cyclic stability and properties of $\mathrm{Cu}$ with highly oriented nanoscale twins. Acta Mater, 2014, 81: 248-257

10 Pan Q, Zhou H, Lu Q, et al. History-independent cyclic response of nanotwinned metals. Nature, 2017, 551: 214-217 